\title{
ESTUDO DA PARTIÇÃO DO FÓSFORO DE ESCÓRIAS DE UM FORNO ELÉTRICO A ARCO ATRAVÉS DA TERMODINÂMICA COMPUTACIONAL*
}

\author{
Mariana Boger Netto ${ }^{1}$ \\ Rodolfo Arnaldo Montecinos de Almeida ${ }^{2}$ \\ Deisi Vieira ${ }^{3}$ \\ Wagner Viana Bielefeldt ${ }^{4}$ \\ Antônio Cezar Faria Vilela ${ }^{5}$
}

\section{Resumo}

O fósforo é um elemento crítico para a qualidade dos aços, tornando a redução dos teores desse elemento uma exigência para a indústria siderúrgica. Uma forma de se medir a eficiência da desfosforação é através do coeficiente de partição do fósforo $\left(\mathrm{Lp}=\% \mathrm{P}_{(\text {escória })} / \% \mathrm{P}_{(\text {aço) })}\right)$. O objetivo principal deste trabalho é avaliar escórias de um forno elétrico a arco através da comparação entre o Lp real com o Lp calculado por simulação termodinâmica, avaliando, também, o comportamento da partição de fósforo com relação à composição química. A partir de dados de composição química de amostras de escória e aço de uma usina siderúrgica, foram feitas simulações termodinâmicas utilizando o software FactSage. Com base nesses resultados, concluiu-se que o cálculo computacional através do software descreve bem o comportamento termodinâmico, mas tende a superestimar o Lp, provavelmente por questões cinéticas e/ou de transferência de massa e calor.

Palavras-chave: Desfosforação; Partição de fósforo; Forno elétrico a arco; Termodinâmica computacional.

\section{STUDY OF SLAGS' PHOSPHORUS PARTITION OF AN ELECTRIC ARC FURNACE THROUGH COMPUTATIONAL THERMODYNAMICS}

\section{Abstract}

Phosphorus is a critical element to the steel's quality, reason why the reduction of phosphorus's contents is a requirement to the steelmaking industry. One method to measure the the dephosphorization efficiency is to calculate the phosphorus partition $\left.\left(\mathrm{L} p=\% \mathrm{P}_{(\text {slag }}\right) \% \mathrm{P}_{(\text {steel) }}\right)$. The main objective of this article is to evaluate slags from an electric arc furnace through the comparison between the real $L p$ and the Lp calculated computationally, also evaluating the phosphorus partition's behavior related to the chemical composition. From slag and steel's chemical composition data, thermodynamic simulations were made using the software FactSage. Based on the results, it was concluded that the computational calculation through the software describes very well the thermodynamic behavior, but it overrates the phosphorus partition, probably because of kinetic considerations and transference of heat and mass.

Keywords: Dephosphorization; Phosphorus partition; Electric arc furnace; Computational thermodynamics.

Graduanda em Engenharia Metalúrgica; Laboratório de Siderurgia (LaSid); UFRGS, RS, Brasil.

Mestre e Engo Metalúrgico, Doutorando; Laboratório de Siderurgia (LaSid), PPGE3M/UFRGS, RS, Brasil.

3 Enga Metalúrgica, Mestranda; Laboratório de Siderurgia (LaSid), PPGE3M/UFRGS, RS, Brasil.

4 Prof. Dr.; LaSid; Depto. de Metalurgia; PPGE3M/UFRGS, RS, Brasil.

5 Prof. Dr.-Ing.;LaSid; Depto. de Metalurgia; PPGE3M/UFRGS, RS, Brasil. 


\section{INTRODUÇÃO}

A redução de teores de fósforo no aço é uma grande exigência que deve ser atendida pela indústria siderúrgica. O fósforo é um elemento crítico na produção de aços, pois é muito sensível à segregação para o contorno de grão, agindo como um poderoso endurecedor por solução sólida e resultando em efeitos prejudiciais como a fragilidade a frio, redução da resistência ao choque e redução de tenacidade e ductilidade [1]. Sendo assim, uma das operações mais importantes no refino dos aços é a remoção do fósforo, chamada desfosforação.

Termodinamicamente, nas condições oxidantes do forno elétrico a arco (FEA), não é possível a remoção de fósforo do banho pela oxidação direta com o oxigênio. $O$ diagrama de Ellingham da figura 1 mostra que o produto formado, $\mathrm{P}_{2} \mathrm{O}_{5}$, não é estável nas temperaturas de processo, podendo ser reduzido imediatamente após a formação e retornar ao banho metálico. Entretanto, sua atividade é reduzida pela adição de $\mathrm{CaO}$ líquido, formando o fosfato tricálcico $\left(3 \mathrm{CaO} \cdot \mathrm{P}_{2} \mathrm{O}_{5}\right.$ ou $\left.\mathrm{C}_{3} \mathrm{P}\right)$, um composto estável nas temperaturas de processo [2]. Por essa razão, escórias de alta basicidade são mais propícias para uma desfosforação mais eficiente.

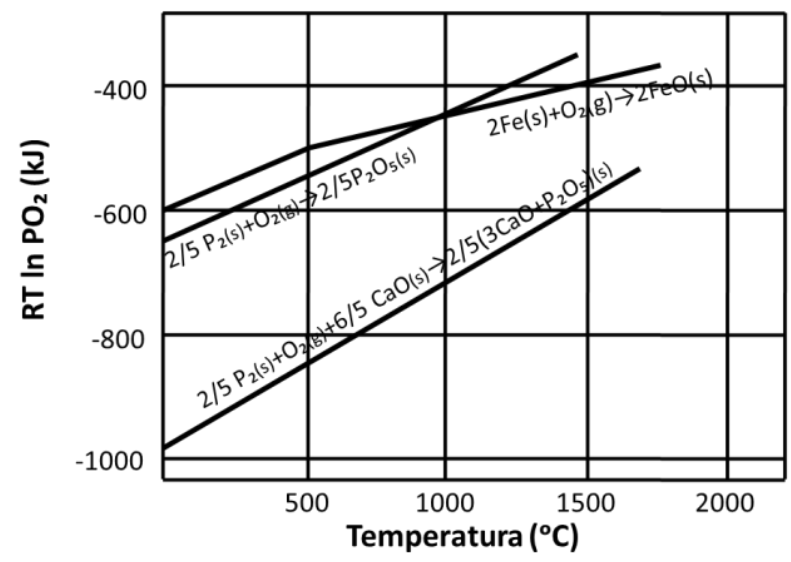

Figura 1: Diagrama de Ellingham simplificado (adaptado de Urban, 2015) [2]

$\mathrm{Na}$ maioria dos casos, escórias utilizadas em desfosforação são básicas e, portanto, saturadas em silicato dicálcico, $2 \mathrm{CaO} . \mathrm{SiO}_{2}$ (também chamado de $\mathrm{C}_{2} \mathrm{~S}$ ). Sabe-se que o silicato dicálcico e o fosfato tricálcico formam uma solução sólida ao longo de uma ampla gama de composição, o $2 \mathrm{CaO} . \mathrm{SiO}_{2}-3 \mathrm{CaO} . \mathrm{P}_{2} \mathrm{O}_{5}\left(\mathrm{C}_{2} \mathrm{SC}_{3} \mathrm{P}\right)$ [3].

Em seu trabalho, Campos demonstrou matematicamente que as reações de desfosforação ocorrem somente na interface entre o metal e a escória [4]. Assim sendo, é necessária uma boa interação entre os mesmos: uma escória favorável à desfosforação deve ser fluida o bastante para permitir que as reações ocorram nessa interface. Além disso, uma boa agitação do banho é indispensável para que mais aço entre em contato com a escória. Portanto, a cinética também se mostra um fator importante no estudo da desfosforação: sabe-se que a cinética da desfosforação é de primeira ordem com respeito à concentração de fósforo no metal e que é controlada, principalmente, pelo transporte de massa na escória $[5,6]$.

Uma forma de se medir a eficiência da desfosforação é através do coeficiente de partição do fósforo (Lp). Esse coeficiente é definido na equação 1, sendo (\%P) o fósforo retido na escória e [\%P] o fósforo existente no banho metálico - quanto maior é o coeficiente de partição, melhor é a desfosforação do banho metálico.

$$
L p=\frac{(\% \boldsymbol{P})}{[\% \boldsymbol{P}]}(1)
$$


A aplicação de métodos computacionais na solução de problemas de termodinâmica tem evoluído consideravelmente à medida que os requisitos sobre a qualidade dos aços se tornam mais rigorosos e os custos de experiências se elevam. Hoje, os programas e bancos de dados disponíveis são ferramentas extremamente úteis para a solução de problemas reais de aciaria e para a previsão de condições recomendáveis para a elaboração e processamento dos aços. [7]

O objetivo principal deste trabalho é a avaliação de dados industriais de um forno elétrico a arco, através da comparação entre o Lp real (calculado a partir da composição química da escória e do aço) com o Lp calculado por simulação termodinâmica, avaliando, também, o comportamento da partição de fósforo com relação à composição química.

\section{MATERIAIS E MÉTODOS}

\subsection{Material}

Neste estudo, foram utilizados dados de composição química da escória e do aço de 172 corridas cedidas por uma unidade do tipo aciaria elétrica. A fim de minimizar fontes de erros, utilizou-se dados de corridas de apenas um padrão de carga fria (fixando, assim, a qualidade da sucata e quantidade de fundentes adicionados no carregamento).

\subsection{Abordagem experimental}

A amostragem foi realizada durante o refino primário do aço de cada corrida, etapa em que ocorre a desfosforação. As amostras de escória tiveram sua composição química determinada através da técnica de espectrometria por fluorescência de raios$\mathrm{x}(\mathrm{XRF})$ e as de aço, através de espectrometria de massa. A tabela 1 mostra a faixa de composição química das amostras recebidas.

Tabela 1: Faixa de composição química da escória estudada (\% em massa)

\begin{tabular}{ccccccc}
\hline$\%$ & $\mathbf{A l}_{\mathbf{2}} \mathbf{O}_{\mathbf{3}}$ & $\mathbf{C a O}$ & $\mathbf{F e O}$ & $\mathbf{M g O}$ & $\mathbf{M n O}$ & $\mathbf{S i O}_{\mathbf{2}}$ \\
\hline Mínimo & 2,93 & 26,73 & 13,22 & 4,59 & 4,26 & 8,86 \\
\hline Máximo & 9,90 & 49,34 & 42,36 & 9,63 & 9,13 & 19,65 \\
\hline
\end{tabular}

Observa-se, nesta tabela, que há uma grande dispersão nos dados industriais. Devido a isso, foram usados filtros na composição química com o intuito de diminuir fontes de erros dos resultados.

Dessa forma, o procedimento experimental foi feito seguindo os passos do fluxograma da figura 2. 


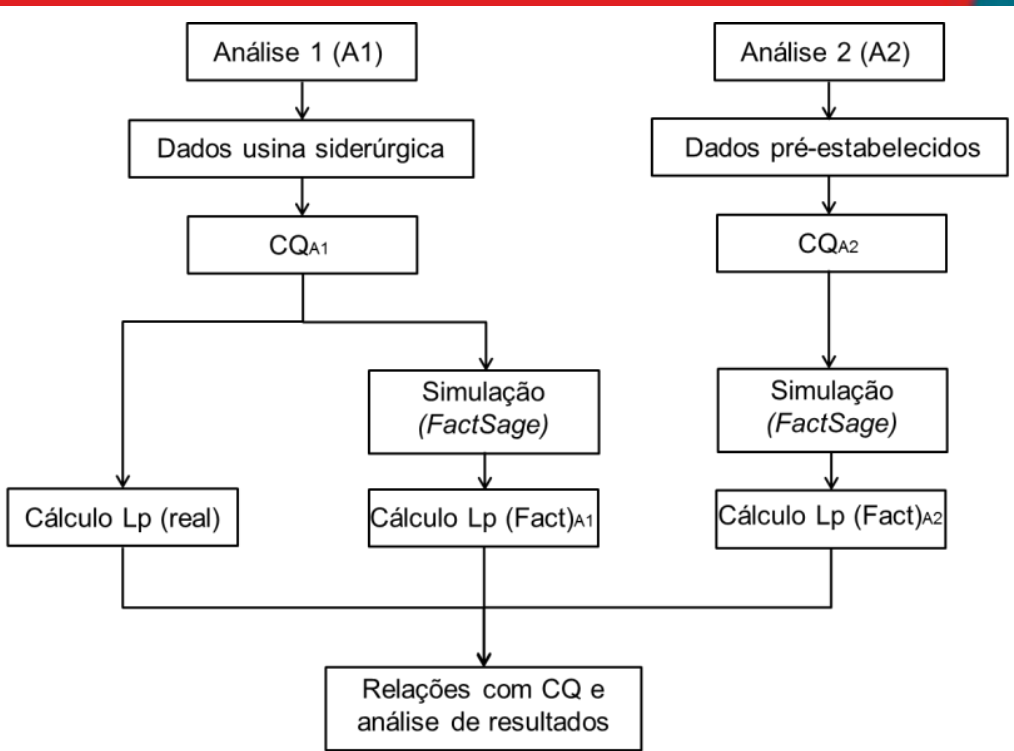

Figura 2: Fluxograma da metodologia aplicada

Na primeira parte da análise, as composições químicas obtidas foram utilizadas no cálculo do coeficiente de partição de fósforo de modo direto (denominado 'Lp real'), através da equação 1. A partir desses dados também foram realizadas simulações termodinâmicas, utilizando o software FactSage, versão 6,4, no módulo Equilib, com os bancos de dados FToxid, FTmisc e GTOX. Este último permite a execução de simulações que implicam na formação de uma fase sólida contendo fósforo, o $2 \mathrm{CaO} . \mathrm{SiO}_{2}-3 \mathrm{CaO} . \mathrm{P}_{2} \mathrm{O}_{5}$. Nas simulações, foi considerado o teor dos principais óxidos contidos na escória $\left(\mathrm{CaO}, \mathrm{FeO}, \mathrm{MgO}, \mathrm{Al}_{2} \mathrm{O}_{3}, \mathrm{MnO}\right.$ e $\left.\mathrm{SiO}_{2}\right)$ e o fósforo total da corrida (correspondente à soma do fósforo contido no aço e na escória, calculados por balanço de massa considerando-se que a proporção entre escória e aço é de 1:20). Além disso, como informações de temperatura não foram recebidas da indústria, foi escolhida a temperatura de $1600^{\circ} \mathrm{C}$. A escolha de uma temperatura implica em erros nos cálculos termodinâmicos e, portanto, nos valores de Lp, pois sabe-se que a as reações de desfosforação são bastante influenciadas pela temperatura. A partir dos resultados das simulações, o Lp também foi calculado através da definição (equação 1) e este foi denominado 'LpFactA1'. Finalmente, os resultados foram relacionados.

A segunda parte da metodologia consiste na elaboração de composições químicas de escórias, variando sempre o teor de $\mathrm{CaO}$ e $\mathrm{SiO}_{2}$ e fixando a basicidade e o teor dos demais óxidos de acordo com a média da composição química das corridas recebidas. Para o estudo sobre a basicidade, foram variados os teores de $\mathrm{CaO}, \mathrm{MnO}$ e $\mathrm{SiO}_{2}$. A composição química destas escórias é apresentada na tabela 2. A temperatura definida para essas simulações também foi de $1600^{\circ} \mathrm{C}$ e foram usados os mesmos bancos de dados. Após isso, o Lp também foi calculado e este foi denominado 'LpFactA2'. Dessa forma, foi possível observar o comportamento do Lp com relação aos principais óxidos (minimizando erros oriundos da influência dos demais e de variações de temperatura), comparar com o comportamento dos dados reais (recebidos da planta) com relação a esses óxidos e, por fim, avaliar o software a respeito da influência de cada óxido no cálculo do Lp.

Tabela 2: Composição química estabelecida para as simulações (\% em massa)

\begin{tabular}{ccccc}
\hline Bas & $\mathrm{Al}_{2} \mathrm{O}_{3}(\%)$ & $\mathrm{FeO}(\%)$ & $\mathrm{MgO}(\%)$ & $\mathrm{MnO}(\%)$ \\
\hline 2,9 & 6,5 & 29 & 6 & 6 \\
\hline
\end{tabular}




\section{RESULTADOS E DISCUSSÃO}

\subsection{Relação entre Coeficientes de Partição de Fósforo}

Primeiramente, foi plotado um gráfico relacionando o Lp real com o Lp FactA1 (figura 3).

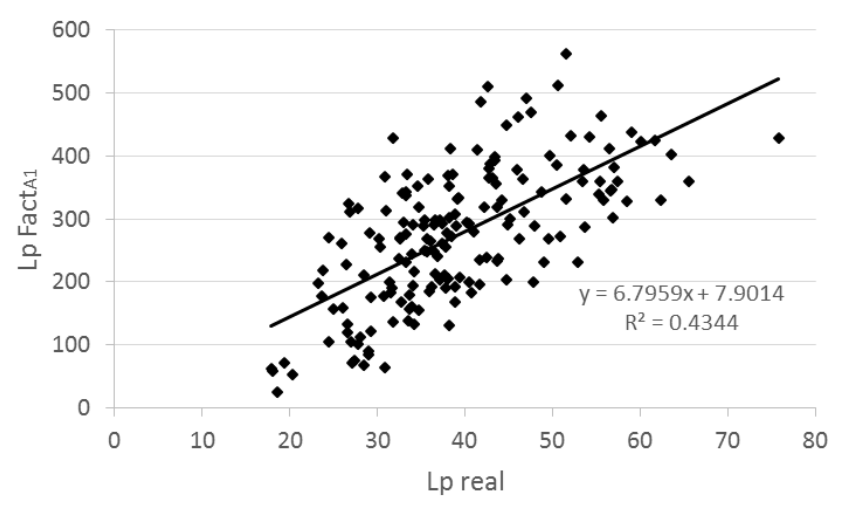

Figura 3: Relação entre Lp real e LpFactA1

A figura 3 mostra que há uma tendência linear entre o $L_{p}$ FactA1 $_{\text {e }}$ o $L p$ real. Porém, analisando as escalas do gráfico, observa-se que o Lp calculado pelo software é sempre maior que o Lp real, e essa diferença cresce conforme o Lp real aumenta. Ou seja, o cálculo computacional através do software FactSage tende a superestimar a partição de fósforo na escória.

Esse resultado é esperado, já que ao realizar o cálculo, o FactSage considera que o sistema tenha atingido um equilíbrio termodinâmico, mas não se pode ter certeza de quão perto dessa condição o processo real está. Basu concluiu em seu trabalho que o Lp real pode variar de 35 a $65 \%$ do valor do equilíbrio, de acordo com a variação da composição química [8]. Já no presente estudo, os valores de Lp real variam de 7,5 a $50 \%$ dos valores de Lp no equilíbrio, com muitos pontos dispersos.

Outro aspecto que deve ser considerado é que o FactSage assume que toda massa do sistema interage. Porém, sabe-se que a desfosforação ocorre somente na interface entre a escória e o aço, e que mesmo em condições de ótima agitação e fluidez, nem toda massa de aço entra em contato com a escória.

A figura 4 mostra a relação entre o fósforo contido na escória dos dados industriais e o fósforo contido na escória calculada pelo software.

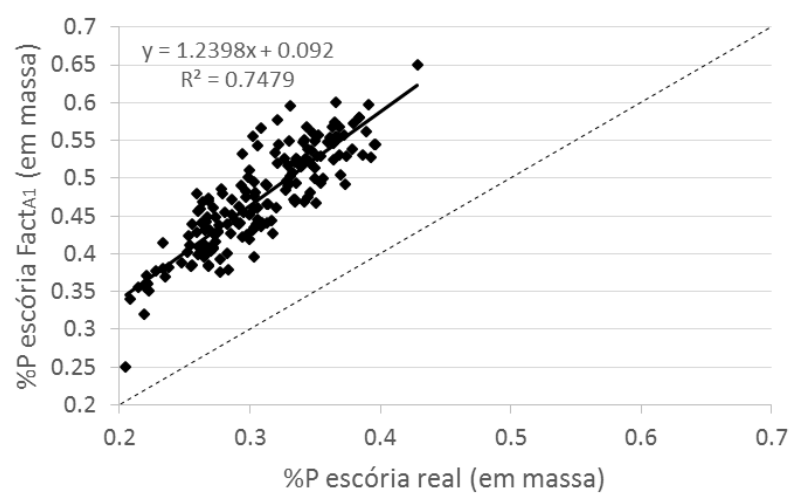

Figura 4: Relação entre \%P na escória dos dados reais e dos resultados do software

Analisando a figura 4, observa-se que há uma relação linear entre a porcentagem de fósforo na escória dos dados recebidos e a obtida computacionalmente. O gráfico mostra que a escória do processo real absorve menos fósforo quando comparado à 
escória obtida pelo software, no equilíbrio. Isso é esperado, já que o software não leva em consideração a cinética no processo de desfosforação e concorda com os altos valores de $L p$ calculados pelo FactSage (Lp FactA1), mostrados na figura 3. A dispersão de dados, considerada baixa, mostra que o comportamento termodinâmico é bem previsto pelo programa. Inserindo-se um fator cinético no cálculo do Lp, é possível que os pontos fiquem mais perto da reta de idealidade.

A figura 5 mostra a relação entre a porcentagem de fósforo no aço dos dados recebidos pela planta siderúrgica e os resultados obtidos pelo software.

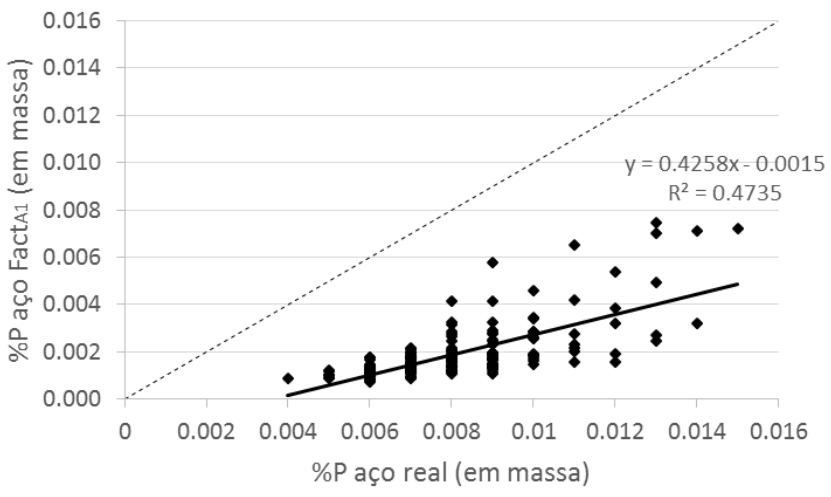

Figura 5: Relação entre \%P no aço dos dados reais e dos resultados do software

A figura 5 mostra que o teor de fósforo no aço do processo real é maior que o teor de fósforo do aço obtido pela simulação termodinâmica, mostrando que, como a escória não pôde absorver todo fósforo que sua capacidade permite, os aços de processos reais têm um teor de fósforo maior que o mostrado pela simulação termodinâmica. Isso pode ocorrer devido à dificuldade de a condição de equilíbrio ter sido alcançada em um processo real. Porém, houve uma alta dispersão dos pontos, que pode ter ocorrido por conta de variações das massas de aço e escória em cada corrida, pela variação de temperatura no momento da amostragem, entre outros fatores operacionais.

\subsection{Relações entre o Lp e a composição química da escória}

Como forma de avaliar o software de simulação e os dados industriais, foi calculado o coeficiente de partição de fósforo de escórias de composição química préestabelecida. A figura 6 relaciona o Lp FactA2 e a basicidade $\left(\% \mathrm{CaO} / \% \mathrm{SiO}_{2}\right)$.

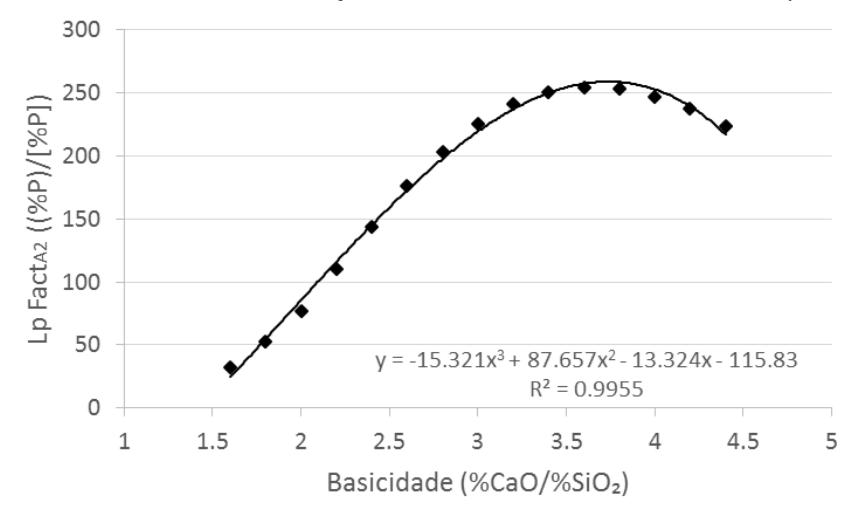

Figura 6: Relação entre o Lp Fact A2 e a basicidade

A figura 6 mostra que o aumento da basicidade leva a um aumento do Lp FactA2, chegando a uma faixa ótima em que o Lp é máximo (entre 3,2 e 4), depois da qual há uma queda nos valores de Lp com o aumento da basicidade. Esse comportamento 
acontece porque a adição de $\mathrm{CaO}$ permite a formação de um composto termodinamicamente mais estável $\left(2 \mathrm{CaO} . \mathrm{SiO}_{2}-3 \mathrm{CaO} . \mathrm{P}_{2} \mathrm{O}_{5}\right)$, que favorece uma melhor desfosforação. Porém, basicidades muito altas representam um excesso de $\mathrm{CaO}$ e pouca oferta de $\mathrm{SiO}_{2}$, óxido também necessário para a formação de $2 \mathrm{CaO} \mathrm{SiO}_{2}-$ $3 \mathrm{CaO} \cdot \mathrm{P}_{2} \mathrm{O}_{5}$. Ocorre, então, a dissolução desse sólido, diminuindo o Lp. A dissolução desse sólido também provoca o aumento da massa líquida da escória. Esse resultado confere com o de outros autores da literatura [2, 9].

As figuras 7 e 8 mostram o Lp real dos dados industriais e o Lp calculado pelo FactSage a partir dos dados industriais relacionados com a basicidade, respectivamente.

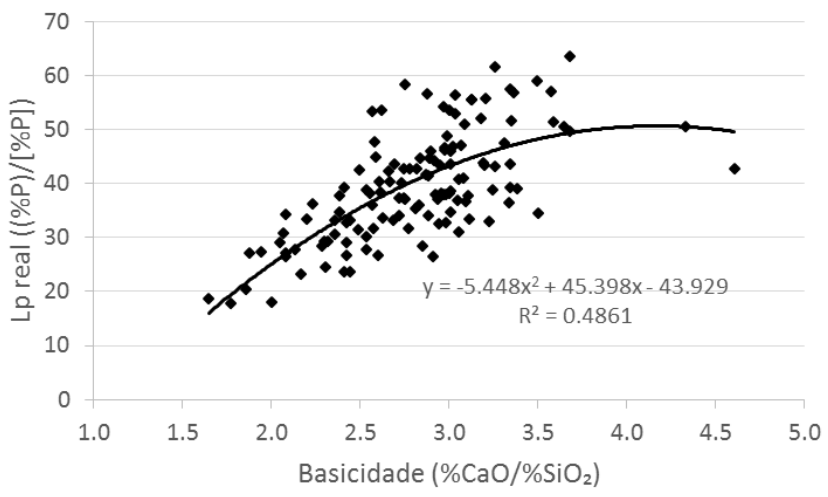

Figura 7: Relação entre Lp real e basicidade

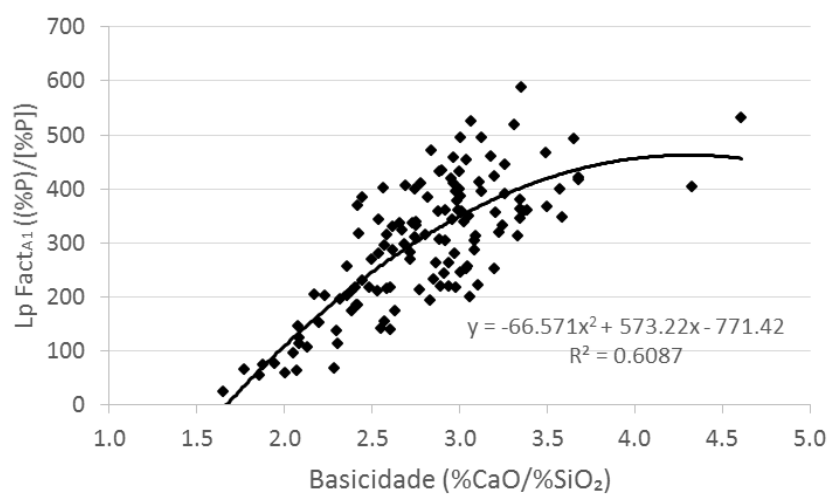

Figura 8: Relação entre LpFact ${ }_{A 1}$ e basicidade

As figuras 7 e 8 mostram que o Lp dos dados industriais teve, em geral, o mesmo comportamento de ascendência com o aumento da basicidade até 3,5, seguido de uma faixa ótima, em que o Lp é máximo. Elas são condizentes também com a figura 6 . Não é muito evidente o decréscimo do Lp em basicidades maiores de 4,0 nas figuras 7 e 8 , mas como só existem duas corridas com essa basicidade, não se pode tirar conclusões. Ao comparar a figura $6 \mathrm{com}$ a figura 8, observa-se que a escala é um pouco diferente: o Lp FactA1 atinge valores maiores no ponto mais alto da curva do que o Lp de composição química dos dados pré-estabelecidos (Lp FactA2). Isso pode ter ocorrido porque, para variar a basicidade e deixar o teor de $\mathrm{CaO}$ constante, foi preciso variar os teores de $\mathrm{MnO}$ e $\mathrm{SiO}_{2}$, ocasionando essa diferença nos valores de Lp.

A figura 9 mostra a relação entre o Lp e o teor de FeO na escória dos dados préestabelecidos.

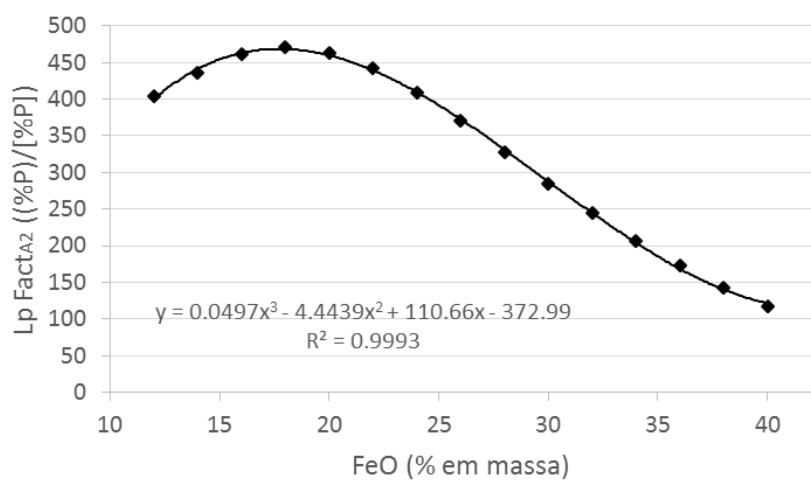

Figura 9: Relação entre o Lp FactA2 e a \%FeO

A relação entre Lp FactA1 e FeO na figura 9 mostra que o Lp aumenta com o aumento de $\mathrm{FeO}$, e depois de atingir um valor máximo (entre 16 a $20 \%$ ), este diminui com o aumento de $\mathrm{FeO}$. Esse comportamento pode ser explicado pelo fato de que enquanto 0 teor de FeO é baixo, o aumento de FeO representa o aumento do potencial de 
oxigênio no banho, levando à oxidação do fósforo e elevando o Lp. A partir de $20 \%$, ocorre um decréscimo no Lp Facta1 na medida em que o teor de FeO aumenta. Segundo alguns autores, quando o teor de $\mathrm{FeO}$ é excessivamente alto, o Lp diminui devido à diluição de óxidos básicos, como o $\mathrm{CaO}[10,11]$.

As figuras 10 e 11 mostram o Lp real e o Lp FactA1 relacionados com o teor de FeO, respectivamente.

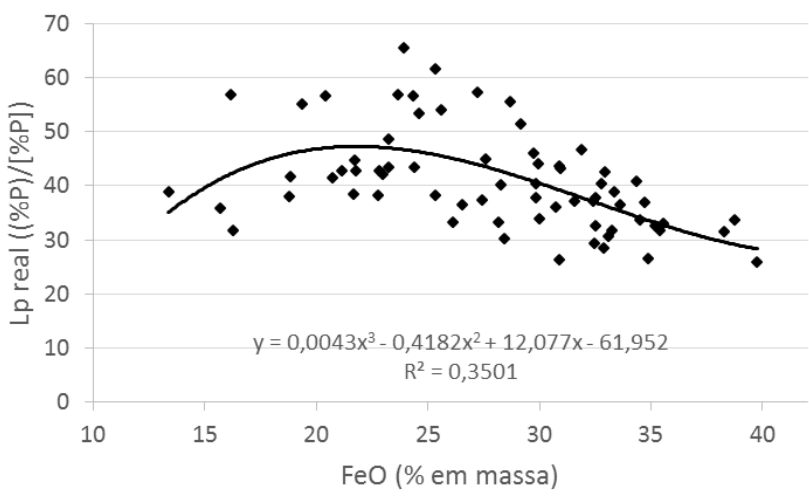

Figura 10: Relação entre o Lp real e a \%FeO

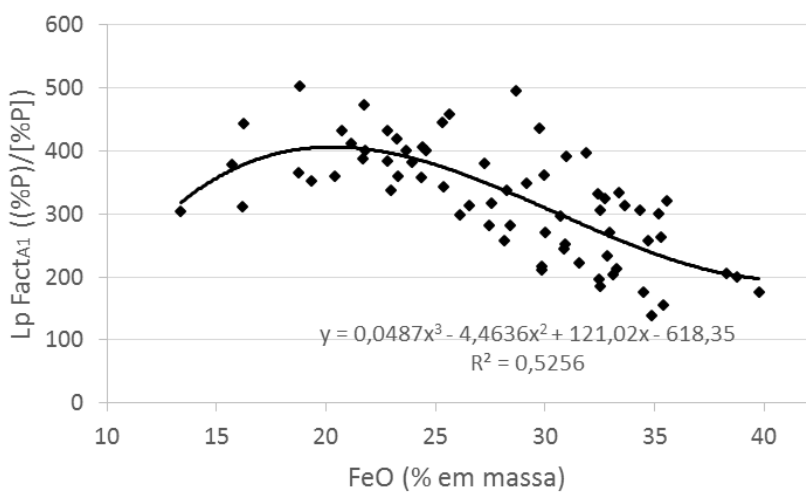

Figura 11: Relação entre o LpFactA1 e a \%FeO

As figuras 10 e 11 mostram que o comportamento do Lp dos dados industriais com relação ao $\mathrm{FeO}$ foi o mesmo, ascendendo em teores pequenos, tendo uma faixa ótima e depois, diminuindo na medida que o teor desse óxido aumenta. Porém, a faixa ótima de FeO para o Lp dos dados industriais se mostrou um pouco mais alta que o Lp FactA2 (vide figura 9), sendo de 17 a $25 \%$. Isso ocorre porque, nas simulações da figura 9 , para variar os teores de $\mathrm{FeO}$, manter a basicidade constante e somar $100 \%$ de massa de escória, foi preciso variar teores de $\mathrm{CaO}$ e $\mathrm{SiO}_{2}$, levando à obtenção de resultados um pouco diferentes das figuras 10 e 11 .

A figura 12 mostra a relação entre o Lp FactA2 e o teor de $\mathrm{Al}_{2} \mathrm{O}_{3}$ na escória dos dados pré-estabelecidos.

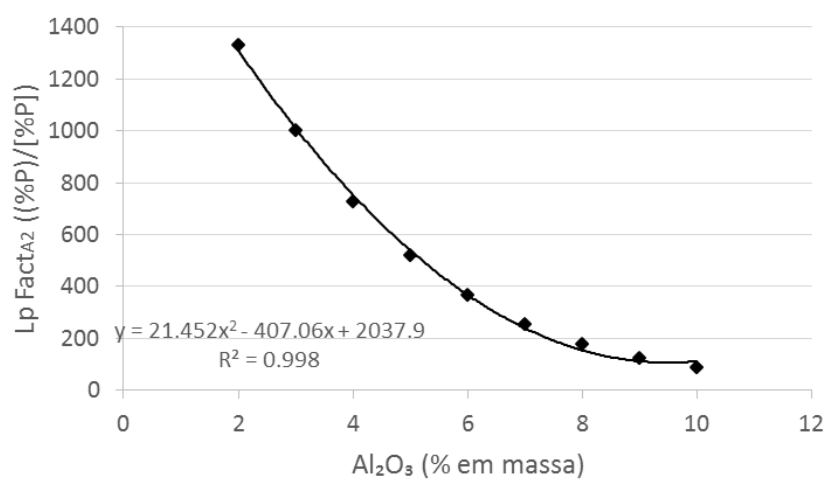

Figura 12: Relação entre o Lp Fact ${ }_{A 2}$ e a $\% \mathrm{Al}_{2} \mathrm{O}_{3}$

A figura 12 mostra que o Lp FactA2 é muito influenciado pela variação do teor de alumina, diminuindo de 1331 a 730 (praticamente à metade) quando o teor de $\mathrm{Al}_{2} \mathrm{O}_{3}$ aumenta de 2 para 4\%. Esse comportamento ocorre porque com a adição desse óxido fluidizante, a fração líquida de escória aumenta e a fração sólida de escória (que contém o fósforo) diminui, promovendo a diminuição de fósforo na escória (ou seja, causando a reversão de fósforo para o banho metálico). Porém, segundo a teoria iônica das escórias, a alumina tem esse comportamento por que ela tira os íons $\mathrm{O}^{-2}$ de outros óxidos, inclusive dos óxidos formados por fósforo, liberando fósforo para o banho 
metálico e diminuindo o $\mathrm{Lp}$ [12]. Esse resultado mostra que o caráter ácido da $\mathrm{Al}_{2} \mathrm{O}_{3}$ predomina sobre o seu caráter fluidizante em escórias com essa composição química. As figuras 13 e 14 mostram o Lp real dos dados industriais e o Lp calculado pelo FactSage a partir dos dados industriais relacionados com 0 teor de $\mathrm{Al}_{2} \mathrm{O}_{3}$, respectivamente.

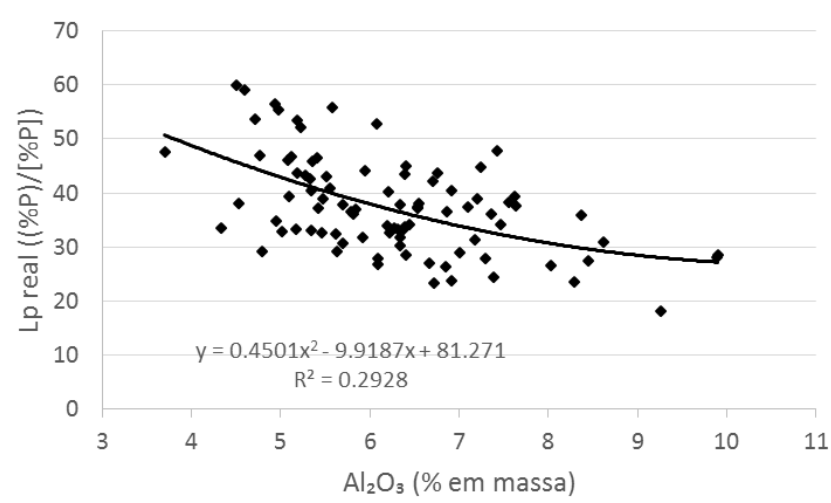

Figura 13: Relação entre o $\mathrm{Lp}$ real e a \% $\mathrm{Al}_{2} \mathrm{O}_{3}$

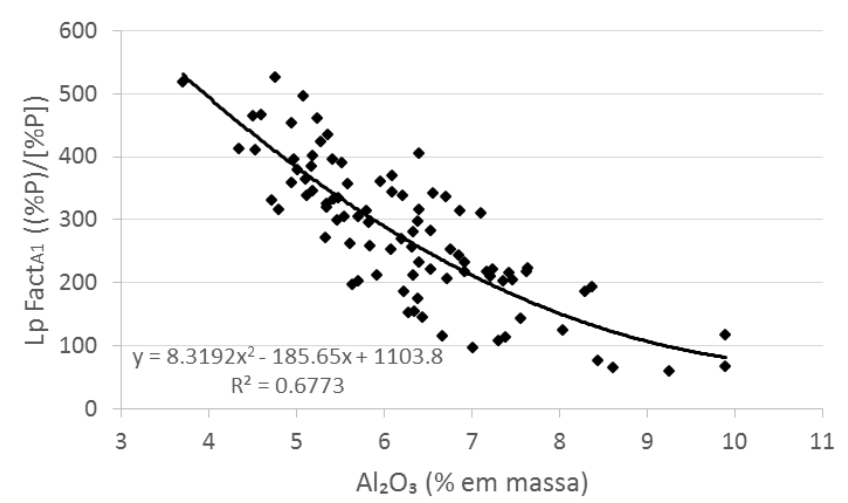

Figura 14: Relação entre o Lp Fact A1 a $\% \mathrm{Al}_{2} \mathrm{O}_{3}$

As figuras 13 e 14 mostram que o Lp dos dados industriais tem o mesmo comportamento decrescente com o aumento no teor de alumina, mas o baixo coeficiente de correlação indica que o efeito desse óxido é menor no cálculo do Lp real do que no cálculo do Lp FactA1. O Lp calculado pelo FactSage a partir dos dados industriais se mostrou muito mais influenciado pela alumina, diminuindo da faixa de 500 para menos de 100 com o aumento de 3,7 a 10\% desse óxido. Contudo, não apresentou valores tão altos quanto aos da figura 12, onde o Lp FactA2 chega a 1331 quando o teor de alumina é de $2 \%$ (não houveram corridas com esse teor de alumina dentro dos filtros utilizados).

A figura 15 mostra a relação entre o Lp e o teor de $\mathrm{MgO}$ na escória dos dados préestabelecidos.

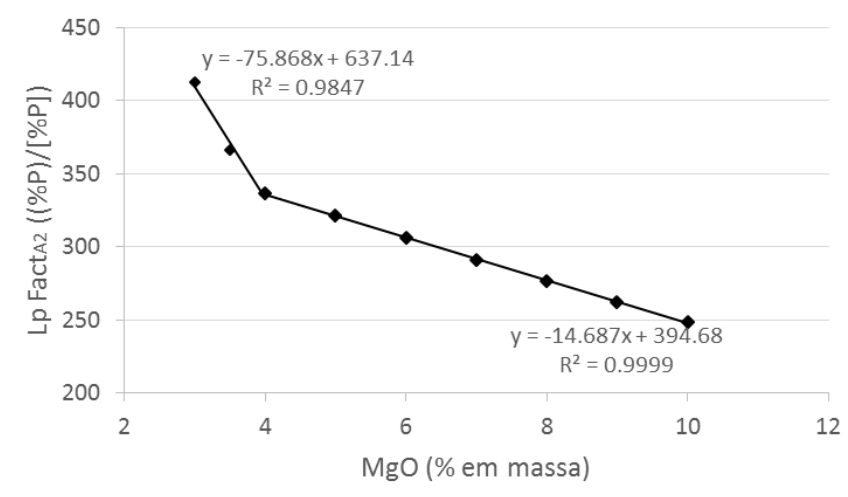

Figura 15: Relação entre o Lp FactA2 e a \%MgO

A figura 15, que relaciona o $\mathrm{Lp}$ FactA2 com o teor de $\mathrm{MgO}$, mostra que também há uma diminuição dos valores de Lp com o aumento desse óxido. Houve uma mudança na declividade da reta causada pela saturação do $\mathrm{MgO}$, que ocorreu entre 3,5 e 4\%. Chen explica, em seu trabalho, que enquanto a escória não foi saturada, a adição de MgO dilui óxidos básicos como $\mathrm{CaO}$, prejudicando a desfosforação. Após a saturação, a adição de $\mathrm{MgO}$ consome o $\mathrm{FeO}$ contido na escória líquida para formação de magnésiowustita, o que aumenta a concentração do $\mathrm{CaO}$ no líquido e eleva o Lp (o intervalo de $\mathrm{MgO}$ estudado por Chen é de $1 \mathrm{a}$ 15\%) [13]. Porém, utilizando o FactSage e no intervalo de $\mathrm{MgO}$ considerado, a adição de $\mathrm{MgO}$ não altera a concentração de $\mathrm{CaO}$ na escória 
líquida após a saturação, e diminui muito pouco o teor de FeO. Consequentemente, a saturação de $\mathrm{MgO}$ somente atenua a taxa de diminuição do Lp.

As figuras 16 e 17 mostram o Lp real dos dados industriais e o Lp FactA1, relacionados com o teor de $\mathrm{MgO}$, respectivamente.

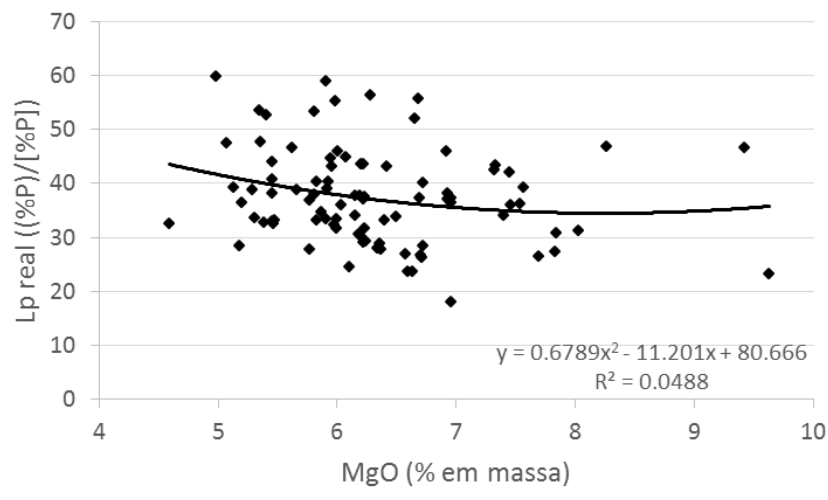

Figura 16: Relação entre o Lp real e a \%MgO

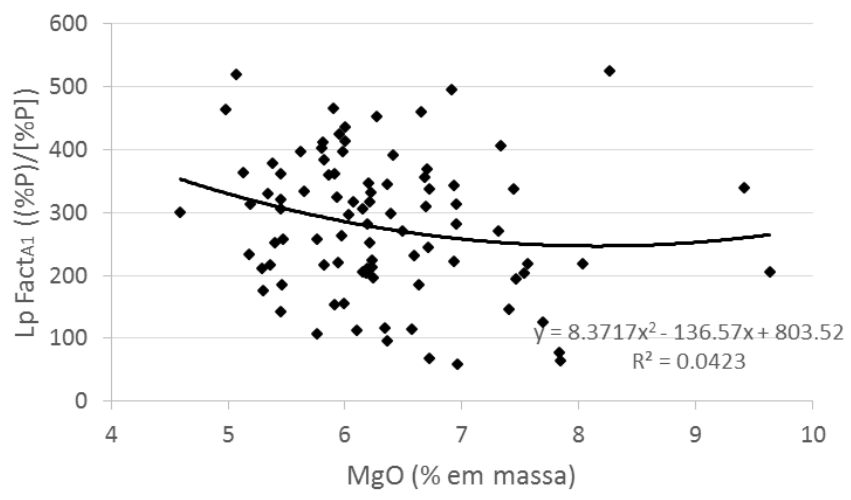

Figura 17: Relação entre o Lp FactA1 e a \%MgO

As figuras 16 e 17 mostram que a dispersão dos pontos da relação entre Lp dos dados industriais e teor de $\mathrm{MgO}$ é muito grande, podendo ter acontecido em função de um ou mais fatores: devido à faixa de $\mathrm{MgO}$, que é muito estreita, ou ao fato de que outros óxidos têm um efeito maior do que o $\mathrm{MgO}$. Portanto, os resultados sobre o MgO são considerados inconclusivos.

\section{CONCLUSÕES}

Para os resultados apresentados nesse trabalho, conclui-se que:

1. O cálculo computacional através do software FactSage tende a superestimar a partição de fósforo e o teor de fósforo na escória, e tende a subestimar o teor de fósforo no aço. Se for empregado diretamente para a previsão desses parâmetros, um fator cinético deverá ser introduzido.

2. Os resultados mostraram que o aço dos dados industriais tem um excesso de fósforo quando comparado com o equilíbrio termodinâmico, mostrando que o sistema não atinge esse equilíbrio.

3. Os resultados obtidos no FactSage sobre o comportamento do Lp com relação aos principais óxidos da escória foram considerados condizentes com a literatura:

- O aumento da basicidade provoca um aumento na partição do fósforo, tendo uma faixa ótima entre 3,2 e 4, em que o Lp é máximo.

- Também existe uma faixa ótima do teor de FeO em que o Lp é máximo, que é de 16 a $20 \%$ desse óxido.

- O aumento no teor de alumina provoca uma diminuição drástica na partição do fósforo da escória.

- O aumento no teor de MgO diminui o Lp, mas a taxa de diminuição muda com a saturação de $\mathrm{MgO}$.

4. A análise de dados industriais com relação à composição química mostrou que o Lp das corridas se comporta como esperado ao variar o teor dos principais óxidos, porém os resultados correspondentes à variação de $\mathrm{MgO}$ se mostraram inconclusivos devido a uma dispersão muito grande dos pontos. 


\section{Agradecimentos}

A aluna agradece ao professor Antônio C. F. Vilela, Coordenador do Laboratório de Siderurgia, pela oportunidade de experiência junto ao laboratório. Ao Professor Wagner Bielefeldt pela orientação, amizade e oportunidade. Aos colegas do Laboratório de Siderurgia pelo apoio e companheirismo. Os autores agradecem à Fundação Luiz Englert e ao CNPq pelo suporte financeiro.

\section{REFERÊNCIAS}

1 SONG, X; YUAN, Z; JIA, J; WANG, D; LI, P; DENG, Z. Effect of Phosphorus Boundary Segregation and Precipitation on Mechanical Properties for Ti-IF Steel after Recrystallization Annealing. Journal of Materials Science \& Technology. 2010; v. 26(9): p. 793-797.

2 URBAN, W; WEINBER, M; CAPPEL, J. Dephosphorization Strategies and Modeling in Oxygen Steelmaking. Iron \& Steel Technology. 2015; p. 91-102.

3 SUITO, H; INOUE, R. Behavior of Phosphorus Transfer from CaO-Fet $\mathrm{O}_{\mathrm{t}}-\mathrm{P}_{2} \mathrm{O}_{5}\left(-\mathrm{SiO}_{2}\right)$ Slag to CaO Particles. ISIJ International. 2006; v. 46 (2): p. 180-187.

4 CAMPOS, V. F. Tecnologia de fabricação do aço líquido. Belo Horizonte: UFMG, 1980. v.1: Fundamentos.

5 MONAGHAN, B. J.; POMFRET, R. J; COLEY, K. S. The Kinetics of Dephosphorization of Carbon-Saturated Iron Using an Oxidizing Slag. Metallurgical and Materials Transactions B. 1998; v.29B: p.111-118

6 NASU, M; MILLS, K. C; MONAGHAN, B. J; KAKOBSSON, A; SEETHARAMAN, S. Effect of Slag/Metal Interfacial Tension on Kinetics of Dephosphorisation. Ironmaking and Steelmaking. 1999; v.26(5): p. 353-357

7 COSTA E SILVA, A. L. Cálculo de Equilíbrio em Aciaria através da Termodinâmica Computacional. Tecnologia em Metalurgia e Materiais. 2006; v.3(1):p.45-52.

8 BASU, S.; LAHIRI, A. K; SEETHARAMAN, S; HALDER, J. Change in Phosphorus during Blowing in a Commercial BOF. ISIJ International. 2007; v.47(5): p. 766-768

9 BASU, S; LAHIRI, A. K.; SEETHARAMAN, S. Phosphorus Partition between Liquid Steel and $\mathrm{CaO}-\mathrm{SiO}_{2}-\mathrm{FeOx}-\mathrm{P}_{2} \mathrm{O}_{5}-\mathrm{MgO}$ Slag Containing 15 to $25 \mathrm{Pct} F e O .2007$; v. 38 B: p. $623-630$

10 BOROVSKY, T; KIJAC,J; DOMOVEC, M. The Slag Composition Influence on the Dephosphorization and on the Lifetime of Eletric Arc Furnace Hearth Refractory Lining. Acta Metallurgica Slovaca. 2010; v.16(3): p. 165-171

11 SASAKI, N; MUKUAWA, S; OGAWA, Y; MIYAMOTO, K. Improvement in Hot-Metal Dephosphorization. Nippon Steel Technical Report. 2013; v. 104: p. 26-32

12 LUCIO, A. Físico Química Metalúrgica. Belo Horizonte: UFMG, 1981. Vol. 2

13 CHEN, C; ZHANG, L; LEHMANN, J. Thermodynamic Modelling of Phosphorus in Steelmaking Slags. High Temperature Materials and Processes. 2013; v.32(3): p.237246. 